\title{
Active clearance technology to maintain chest tube patency: Practical, innovative, unproven
}

\author{
Nicholas D. Andersen, MD
}

\footnotetext{
From the Division of Cardiovascular and Thoracic Surgery, Department of Surgery, Duke University Medical Center, Durham, NC.

Disclosures: Author has nothing to disclose with regard to commercial support.

Received for publication Nov 5, 2015; accepted for publication Nov 6, 2015; available ahead of print Dec 9, 2015 Address for reprints: Nicholas D. Andersen, MD, Division of Cardiovascular and Thoracic Surgery, Duke University Medical Center, Box 3443, Durham, NC 27710 (E-mail: Nicholas.andersen@ duke.edu).

J Thorac Cardiovasc Surg 2016;151:839-40

$0022-5223 / \$ 36.00$

Copyright (C) 2016 by The American Association for Thoracic Surgery

http://dx.doi.org/10.1016/j.jtcvs.2015.11.008
}

Large-bore chest tubes remain the sine qua non of the cardiothoracic surgeon's arsenal for evacuating blood from the chest. Chest tube clogging can be a frequent and vexing problem, however, leading to inadequate mediastinal or pleural drainage and occasionally resulting in serious complications such as cardiopulmonary compromise or reoperation. To combat this problem, a novel chest tube with a guidewire-based clearance mechanism, dubbed "active tube clearance" (ATC), was recently developed and has shown promise in preclinical studies. ${ }^{1,2}$ As described in their article in this issue of the Journal, to validate this technology further in a real-world clinical setting, Sirch and colleagues ${ }^{3}$ placed a single anterior mediastinal ATC chest tube in all patients undergoing cardiac surgery during a 15-month period at a cardiac surgical center in Germany and implemented a standardized protocol for tube clearance to maintain patency of the ATC chest tube during the first 24 hours. Additional standard chest tubes were placed and managed at the discretion of the operative surgeon and were not controlled for in the protocol. Outcomes were then compared before and after use of the ATC chest tube and again after discontinuation of use. The key findings of Sirch and colleagues ${ }^{3}$ were that the ATC chest tube use reduced "retained blood syndrome" by $43 \%$ and atrial fibrillation by $33 \%$; however, a closer examination of these data reveals several incongruent findings that limit the strength of the conclusions.

First, contrary to the title of the article, Sirch and colleagues $^{3}$ did not evaluate or measure retained mediastinal blood to prove that the device achieved the stated goal. Instead, total chest tube drainage was measured; but it was paradoxically lower with use of the ATC chest tube, in contrast to the preclinical studies in which the ATC chest tube led to increased chest drainage in a bleeding model relative to standard chest tubes. ${ }^{1,2}$ Thus whether the ATC chest tube actually improved drainage and reduced retained mediastinal blood remains unclear and confounds the remainder of the results and mechanistic explanations.

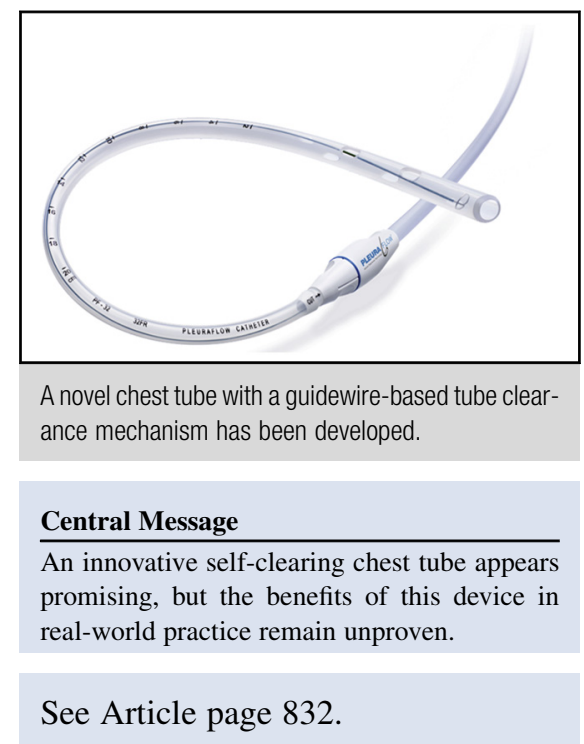

Instead of measuring retained blood, the study hinges on a previously undefined and unvalidated end point, "retained blood syndrome," which is not a syndrome at all but simply a composite of reinterventions that may or may not be related to retained mediastinal blood. These reinterventions included reexploration for bleeding, pericardial drainage procedures, and pleural drainage procedures for effusion or air. Results showed there was no reduction in any of these isolated interventions after ATC chest tube use; the composite end point was statistically reduced, however, primarily because of a lower number of pleural interventions for effusion or pneumothorax. Given that all opened pleural cavities reportedly underwent chest tube placement at the time of operation, the mechanism whereby improved anterior mediastinal drainage within the first 24 hours of surgery leads to reduced effusion or air in an unopened pleural cavity is speculative and requires further objective validation. Additional information regarding the nature of the pleural collection, (simple effusion, bloody effusion, air), timing of intervention (delayed or immediate), and the number, location, and management of all other chest tubes is also required for full interpretation of these results.

Lastly, the unadjusted rate of retained blood syndrome remained constant during the ensuing 3 months after discontinuation of ATC chest tube use $(11.3 \%$ vs $17.6 \%$; $P=.07$ by Fisher's exact test). Together with the concerning finding that the rate of retained blood syndrome did not correlate with previously validated risk factors for bleeding, 
such as procedural complexity, urgency status, or reoperation, the validity of this newly defined end point and its relationship to retained mediastinal blood is questionable. The secondary finding of reduced atrial fibrillation is perhaps the most plausible evidence of benefit from improved mediastinal drainage as a result of reduced atrial irritation; however, the unadjusted rates of atrial fibrillation also remained constant after discontinuation of ATC use $(20.3 \%$ vs $23.9 \% ; P=.38$ by Fisher's exact test). Together, these findings question whether the reductions in chest tube drainage, retained blood syndrome, and atrial fibrillation observed with time were due not to ATC chest tube usage but instead to era effect and other temporal practice changes not controlled for in this nonrandomized analysis.

Despite these concerns, the ATC chest tube technology appears practical and innovative, and it will likely find utility in certain clinical settings, such as in patients at high risk of bleeding or those in whom mediastinal or pleural chest tubes are expected to remain in place for long periods. The data presented thus far supporting the promotional claims of efficacy of this product in all cardiac surgical patients ${ }^{4}$ appear premature, however, and they should be considered hypothesis generating at this time. Given a cost of $\$ 395$ per chest tube, ${ }^{4}$ a well-designed prospective, randomized clinical trial with appropriate clinical end points and cost analysis is recommended before this technology is widely adopted by the cardiac surgical community and applied indiscriminately to all patients.

\section{References}

1. Arakawa Y, Shiose A, Takaseya T, Fumoto H, Kim HI, Boyle EM, et al. Superior chest drainage with an active tube clearance system: evaluation of a downsized chest tube. Ann Thorac Surg. 2011;91:580-3.

2. Shiose A, Takaseya T, Fumoto H, Arakawa Y, Horai T, Boyle EM, et al. Improved drainage with active chest tube clearance. Interact Cardiovasc Thorac Surg. 2010; 10:685-8.

3. Sirch J, Ledwon M, Püski T, Boyle EM, Pfeiffer S, Fischlein T. Active clearance of chest drainage catheters reduces retained blood. J Thorac Cardiovasc Surg. 2016; 151:832-8.

4. ClearFlow [Internet]. Anaheim (CA): ClearFlow; c2015. Available at: http://www. clearflow.com. Accessed November 2015. 